Communications in Physics, Vol. 23, No. 2 (2013), pp. 155-161

\title{
THE INFLUENCE OF THE SELF-FOCUSING EFFECT ON THE OPTICAL FORCE ACTING ON DIELECTRIC PARTICLE EMBEDDED IN KERR MEDIUM
}

\author{
HOANG VAN NAM \\ Council of Science and Technology, Ha Tinh, Vietnam \\ CAO THANH LE \\ Ha Tinh University \\ No. 447, 26/3 Road, Dai Nai, Ha Tinh City, Ha Tinh, Vietnam \\ HO QUANG QUY \\ Academy of Military Science and Technology \\ 17 Hoang Sam Street, Nghia Do, Cau Giay, Hanoi, Vietnam \\ Received 30 January 2013; revised manuscript received 31 May 2013 \\ Accepted for publication 10 April 2013
}

\begin{abstract}
The influence of the self-focusing effect arised from Kerr effect on the optical force acting on the dielecric particle embedded in the Kerr medium, which is irradiated by the Gaussian beam, is proposed to concern. The expressions of the optical forces with the nonlinear refractive index and nonlinear focal length are derived. Using them, the distribution of the optical forces in the trapping region of the optical tweezer is simulated and discussed for same distinguished case of the Kerr medium with different nonlinear coefficients. The results show that the stabe region of the optical tweezer depends on the nonlinear coefficient of refractive index. Moreover, the stable region could be brokendown with a critical value of the nonlinear coefficient of refractive index of the surrounding medium irradiated by Gaussian laser pulse described by given parameters as intensity, duration and radius of beam waist.
\end{abstract}

\section{INTRODUCTION}

The optical tweezer used CW laser beam is used to trap the micro dielectric particle, which is embedded in a medium as gas, fluid [1-8]. Up to now, the optical trap using pulsed Gaussian beam and the optical trap using counter-propagating pulsed Gaussian beams has been paid attention to trap the nanoparticle [9-11]. Unfotunately, in the mentioned works, there was attention to thermal effect of the medium only (the Brownian motion), but not to other effect as random or Kerr. In work [12], the optical force acting on dielectric particle embedded in the random medium, which causes the partial coherence of laser beam is discussed. In work [13], the Kerr effect appeared in the medium or inside the particle, which is irradiated by intense laser pulse is concerned for the optical tweezer. However, in this work, the self-focusing of the laser beam, which is an important effect relating to Kerr effect, has been not concerned. 
Therefore, in this paper, the influence of the both effects, the Kerr in medium and the self-focusing of the laser beam in optical tweezer on the distribution of optical force acting on dielectric nanoparticle are investigated. This article is organized as follows: in Sec. 2 we derive the expressions of the optical forces concerning self-focusing, which is arised from the Kerr effect in the Kerr medium irradiated by the Gaussian pulse; in Sec.3 we present the influence of the nonlinear coefficient of refractive index on the optical forces and discuss about the stability of the optical tweezer.

\section{OPTICAL FORCES}

As a example, we consider an optical tweezer to trap a dielectric nano particles in the Kerr medium (see Fig. 1). A pulsed plane laser TEM $_{00}$ beam focused by a lens, and the electric field can be approximately expressed by [7]:

$$
\begin{aligned}
\vec{E}(\rho, z, t)= & \hat{x} E_{0} \frac{i k W_{0}^{2}}{i k W_{0}^{2}+2 z} \exp \left[-i\left(k(z)-\omega_{0} t\right)\right] \\
& \times \exp \left[-i \frac{2 k z \rho^{2}}{\left(k W_{0}^{2}\right)+2 z^{2}}\right] \times \exp \left[-\frac{\left(k W_{0}^{2}\right)^{2} \rho^{2}}{\left(k W_{0}^{2}\right)^{2}+4 z^{2}}\right] \\
& \times \exp \left[-(t-z c)^{2} / \tau^{2}\right],
\end{aligned}
$$

as a Gaussian beam, where $W_{0}$ is the radius of the beam waist at $z=0, \rho$ is the radial coordinate, $\hat{x}$ is the unit vector of the polarization along the $x$ direction, $k=2 \pi / \lambda$ is the wave number, $\omega_{0}$ is the carrier frequency, $\tau$ is the pulse duration, $c$ is the light speed.

Assuming that this pulse causes the Kerr effect in the medium surrounding the particle. So after propagating through medium the Gaussian beam will be self-focused by the nonlinear lens of focal length (see Fig. 1) which is approximately given by [14]:

$$
f_{n l}\left(P, n_{n l}\right)=W_{0}^{2} \sqrt{\left(\frac{\pi n_{2}}{2 n_{n l} P}\right)}
$$

where $n_{2}, n_{n l}$ are the linear refractive index and nonlinear refractive coefficient of the Kerr medium, respectively, and $P=2 \sqrt{2} U /\left[(\pi)^{3 / 2} W_{0}^{2} \tau\right]$ is the total power of the input Gaussian beam.

It can be considered that the Kerr medium with distance of $z^{\prime}$ plays as the thin lens with focal length of $f_{n l}$. Such that, after propagating through the Kerr medium, the Gaussian beam is modified and its waist radius, $W_{0}$ is modified into [15]:

$$
W_{0}^{\prime}\left(P, n_{n l}\right)=M\left(P, n_{n l}\right) W_{0},
$$

where $M\left(P, n_{n l}\right)=M_{r} / \sqrt{1+r^{2}}, r=z_{0} /\left(z^{\prime}-f_{n l}\right), M_{r}=\left|f_{n l} / z^{\prime}-f_{n l}\right|, z_{0}=\pi W_{0}^{2} / \lambda$ is the Rayleigh range, $z^{\prime}$ is the distance from beam waist to thin lens, which has minus sign for considered optical tweezer in Fig. 1. 
From (1), (3) and the definition of the Pointing vector, we can readily obtain the intensity distribution for the redistributed pulse in the tweezer as follows:

$$
I(\rho, z, t)=\frac{P}{1+4 \tilde{z}^{2}} \exp \left[-\frac{2 \tilde{\rho}^{2}}{1+4 \tilde{z}^{2}}\right] \times \exp \left[-2\left(\tilde{t}-\frac{\tilde{z} k W_{0}^{\prime 2}}{c \tau}\right)^{2}\right]^{2}
$$

where $\tilde{z}=z / k W_{0}^{\prime 2} \tilde{\rho}=\rho / W_{0}^{\prime}$ and $\tilde{t}=t / \tau$.

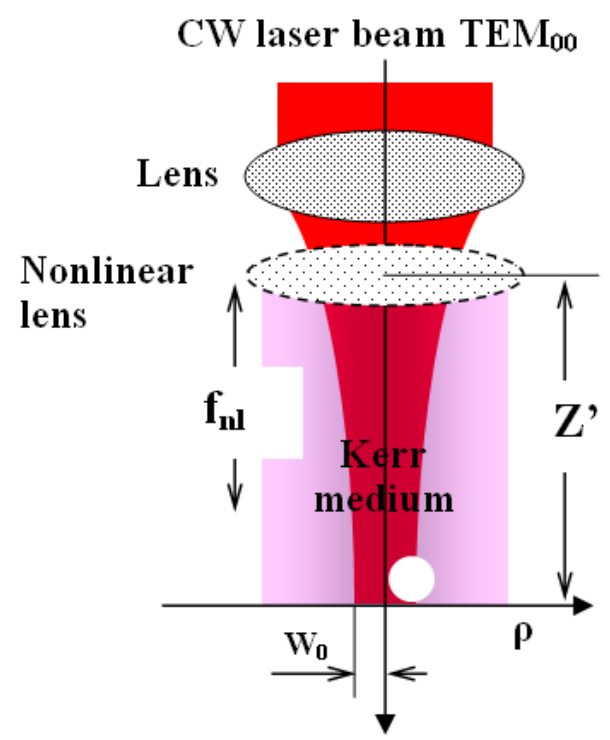

Fig. 1. Sketch of optical tweezer with Kerr medium.

For simplicity, we assume that the radius $(a)$ of the linear particle (considering the particle is not sensitive to Kerr effect) is much smaller than the wavelength of the laser (i.e., $a<<\lambda$ ), in this case we can treat the dielectric particle as a point dipole. We also assume that the refractive index of the dielectric particle is $n_{1}$ and $n_{1}>>n_{2}$. This is the necessary condition for trapping operation of the tweezer using Gaussian beam [16].

The medium is Kerr, then its refractive index will be

$$
n_{m}(\rho, z, t)=n_{2}+n_{n l} I(\rho, z, t)
$$

and the relative refractive index is given by

$$
m_{m}(\rho, z, t)=n_{1} / n_{m}(\rho, z, t)
$$

Using (6) and as shown in Refs. [7, 8], we have the scattering cross sections $(\alpha)$ and polarizabilities $(\beta)$ as follows:

$$
\alpha_{m}(\rho, z, t)=\left(\frac{128 \pi^{5} a^{6}}{3 \lambda^{4}}\right)\left[\frac{\left(m_{m}(\rho, z, t)^{2}-1\right)}{\left(m_{m}(\rho, z, t)^{2}+2\right)}\right]^{2}
$$


and

$$
\beta_{m}(\rho, z, t)=4 \pi n_{m}^{2}(\rho, z, t) \varepsilon_{0} a^{3} \frac{\left(m_{m}(\rho, z, t)^{2}-1\right)}{\left(m_{m}(\rho, z, t)^{2}+2\right)}
$$

To show influence of self-focusing effect on the optical force, as example for simplicity, we present here expressions of the longitudinal force along the beam axis (at $\rho=0$ ) and transverse gradient force in the specimen plane (at $z=0$ ) when $t=0$ (at moment laser pulse reaches peak).

As shown in previous works $[9,10,13,17]$, the transverse gradient force is reduced to:

$$
\vec{F}_{\text {grad }, \rho}(\rho, 0,0)=-\hat{\rho} \frac{2 \beta_{m}(\rho, 0.0) I(\rho, 0,0) \tilde{\rho}}{c n_{m}(\rho, 0,0) \varepsilon_{0} W_{0}^{\prime}\left(P, n_{n l}\right)}
$$

and the longitudinal force is given by:

$$
\begin{aligned}
\vec{F}_{z} & =-\vec{z} \frac{2 \beta_{m}(0, z, 0) I(0, z, 0)}{n_{m}(0, z, 0) \varepsilon_{0} c k w_{0}^{\prime}\left(P, n_{n l}\right)}\left[\frac{\tilde{z} k^{2} w_{0}^{\prime}\left(P, n_{n l}\right)}{c^{2} \tau^{2}}+\frac{2 \tilde{z}\left(1+4 \tilde{z}^{2}\right)}{\left(1+4 \tilde{z}^{2}\right)^{2}}\right] \\
& +\vec{z} \frac{n_{m}(0, z, 0)}{c} \alpha_{m}(0, z, 0) I(0, z, 0)
\end{aligned}
$$

The general Exps. (9) and (10) describe the redistribution of gradient force in speciment plane $(\rho)$ and longitudinal force in beam axis $(s)$ caused by both effects: self-focusing $(d)$ and refractive index's nonlinearity $\left(n_{n l}\right)$. If $f_{n l}=\infty$, from (3) results $M(P, n l)=1$ or $W_{0}^{\prime}\left(P, n_{n l}\right)=W_{0}$, i.e. the self-focusing effect is ignored, Exps. (9) and (10) coincide with Exps. (9) and (10), respectively, in the our previous work [13].

\section{SIMULATED DISTRIBUTION OF OPTICAL FORCES}

In following numerical simulation we choose parameters as: $U=10 \mu J, W_{0}=2 \mu m, \tau=$ $10 \mathrm{~ns}, \lambda=0.53 \mu \mathrm{m}$ (for Gaussian pulse); the refractive index of the glass particle with radius $a=20 \mathrm{~nm}$ is $n_{1}=1.592$; this particle is embedded on water of refractive index $n_{2}=1.332$. Moreover, we considered the water is dissolved by the nonlinear material, so their nonlinear coefficient should be changed.

The optical gradient force $F_{\text {grad, } \rho}$ and $F_{z}$ are calculated by expression (9) and (10) in the ranges: $\rho=(-2 \div 2) \mu \mathrm{m}$ and $z=(-5 \div 5) \mu \mathrm{m}$ (consider the beam waist of pulsed Gaussian beam located in the traping plane $z=0$ and the beam axis goes through point $\rho=0$ ), respectively. The distributions of the gradient force in the specimen plane and of the longitudinal force in the beam axis are simulated and shown in Fig. 2 and Fig. 3, respectively.

It can be seen that, if the medium is not Kerr medium or the nonlinear refractive coefficient of it is small $\left(n_{n l}=1 \times 10^{-11} \mathrm{~cm}^{2} / \mathrm{W}\right)$, the particle will be trapped in a certain stable region with a radial radius of about $W_{0} / 2$ in the speciment plane (Fig. 2a) and a length of about $3 \mu \mathrm{m}$ along beam axis on the medium (Fig. 3a). If the nonlinear coefficient increases, the optical force decreases and the stable region is larger. Especially, if the nonlinear coefficient is large enough, inside the trapping region appears a small unstable region, where the particle moves along the direction of the force (Figs. 2b, 2c, $3 \mathrm{~b}$, and $3 \mathrm{c})$, because of the changing sign of $\beta_{m}$. This phenomenon appears due to the Gaussian distribution of the laser intensity, i.e. the intensity in the center of the laser beam is larger than one in the edge. 


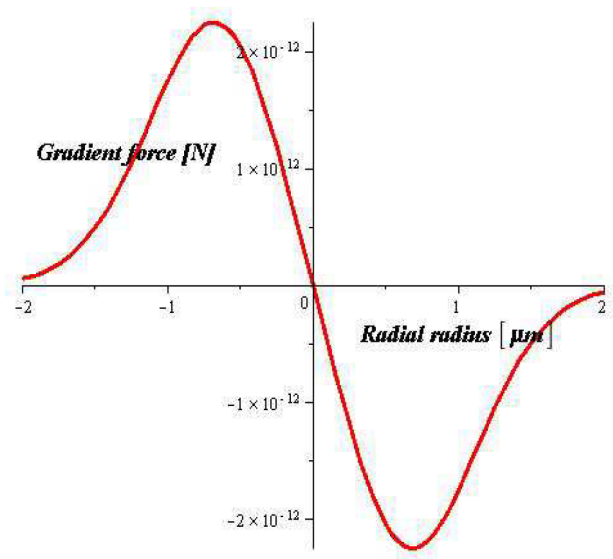

a)

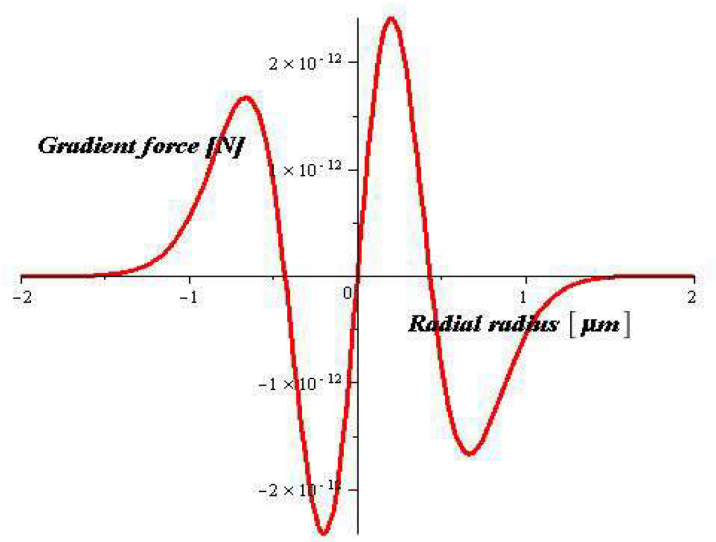

c)

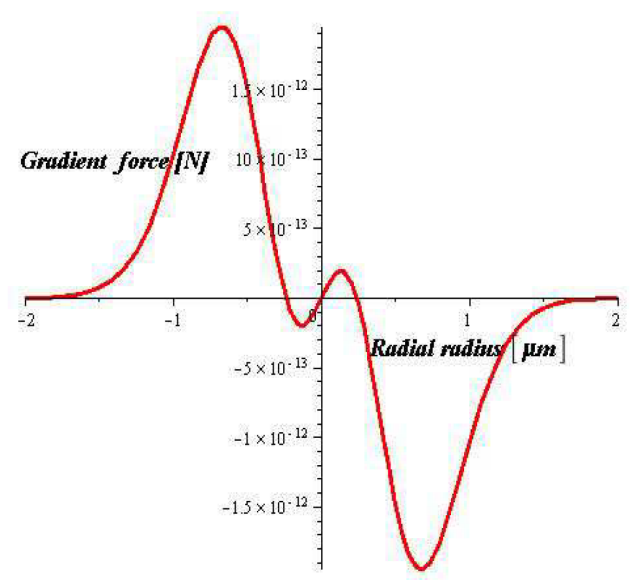

b)

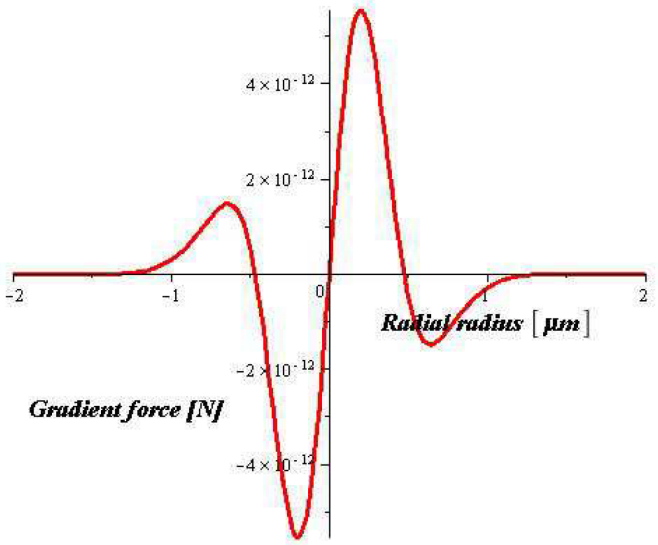

d)

Fig. 2. Distribution of gradient force $[\mathrm{N}]$ on radical radius $[\mu \mathrm{m}]$ with different value of the nonlinear coefficient of the medium: a) $\mathrm{n}_{n l}=1 \times 10^{-11} \mathrm{~cm}^{2} / \mathrm{W}$; b) $n_{n l}=3 \times 10^{-11} \mathrm{~cm}^{2} / \mathrm{W}$; c) $n_{n l}=5 \times 10^{-11} \mathrm{~cm}^{2} / \mathrm{W} ;$ d) $n_{n l}=7 \times 10^{-11} \mathrm{~cm}^{2} / \mathrm{W}$.

The unstable region is more and more large with increasing of the nonlinear refractive coefficient (Fig. 2d, Fig. 3d). This phenomenon can be explained by the decreasing of the relative refractive index $m_{m}$, consequently, reducing the refraction of laser rays through the particle. It means the trapping forces are reduced.

If the nonlinear coefficient is too large, because of the Kerr effect the Gaussian beam will be self-focused, since that, the refractive index of the medium inside defined stable region will be larger than that of particle. In this case, $m_{n}<1$ (i.e. $\alpha_{m}, \beta_{m}<0$ ), consequence sign $\vec{F} \equiv \operatorname{sign} \vec{\rho}$, where $\vec{\rho}$ is the radial variance of particle [14], the particle will be pushed ouside the centre of tweezer (Figs. 2c, 2d and Figs. 3c, 3d), and the tweezer will be brokendown. 


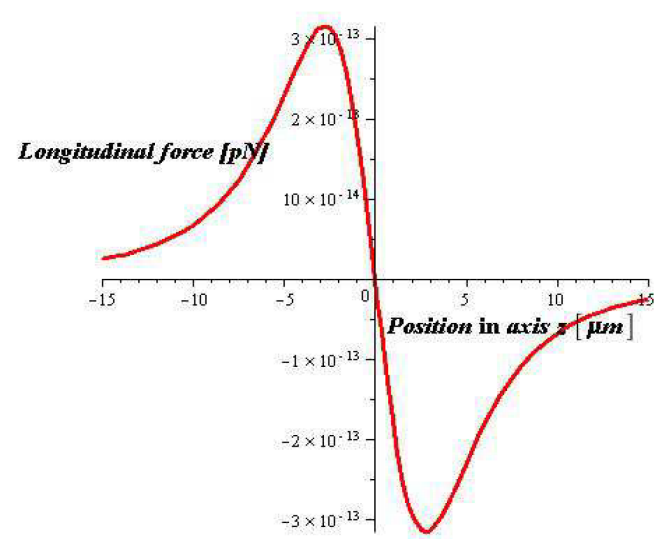

a)

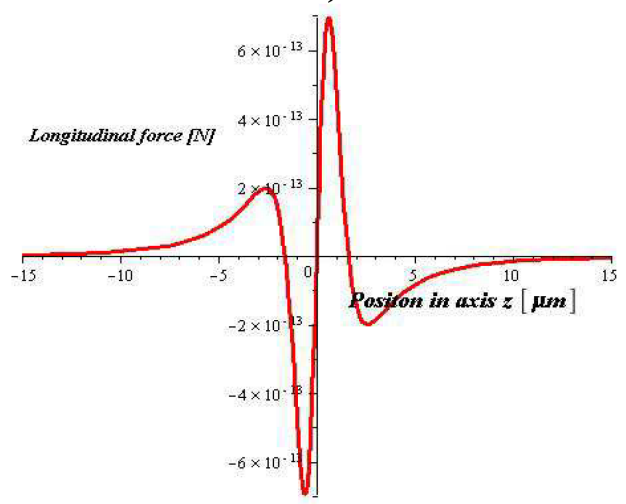

c)

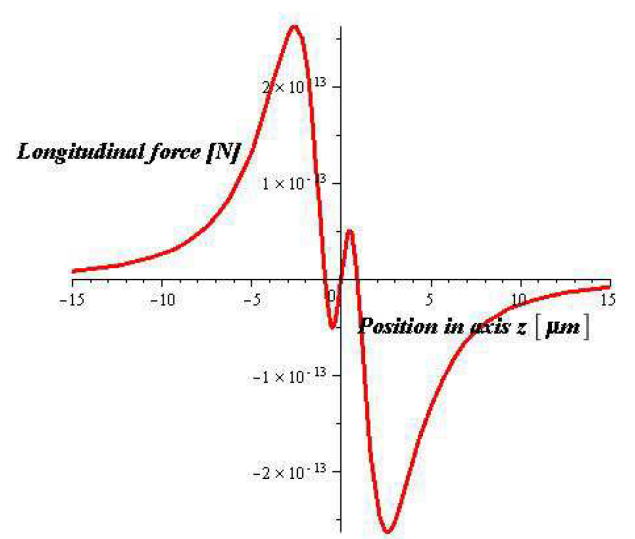

b)

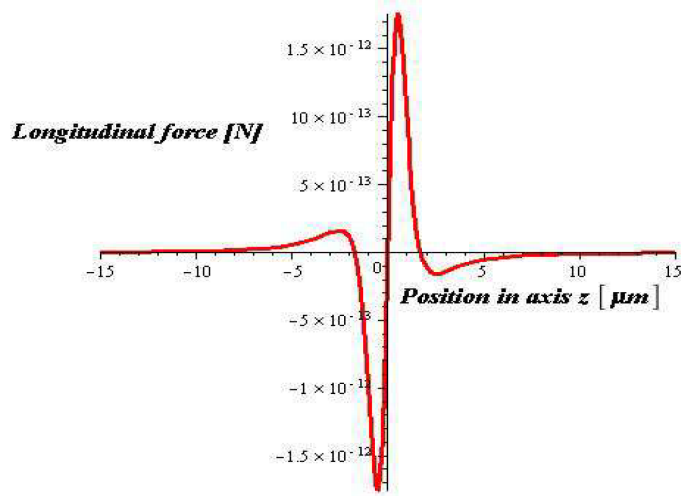

d)

Fig. 3. Distribution of longitudinal force $(N)$ on beam axis $(\mu \mathrm{m})$ with different value of the nonlinear coefficient of the medium: a) $n_{n l}=1 \times 10^{-11} \mathrm{~cm}^{2} / \mathrm{W}$; b) $n_{n l}=3 \times 10^{-11} \mathrm{~cm}^{2} / \mathrm{W}$; c) $n_{n l}=5 \times 10^{-11} \mathrm{~cm}^{2} / \mathrm{W}$; d) $n_{n l}=7 \times 10^{-11} \mathrm{~cm}^{2} / \mathrm{W}$.

From Exps. (2), (5), (9) and (10), we can see that, the distribution of optical forces and consequence, the stable region depend not on $n_{2 n}$ only, but also on energy $U$, beam waist $W_{0}$ and duration $\tau$ of the pulsed Gaussian beam.

\section{CONCLUSION}

The distribution optical forces depend on the nonlinear refractive coefficient of the surrounding medium. If the medium is a nonlinear one (the particle is linear), the Kerr effect and the relating self-focusing effect reduce the trapping forces, so far brokendown the trapping. The dimension of the stable region depend not on the nonlinear coefficient $n_{n l}$ only, but also on the total energy $U$, beam waist $W_{0}$ and duration $\tau$ of the laser pulse and other parameters of the particle and medium. As shown above, there is a collection of parameters: $n_{n l}=7 \times 10^{-11} \mathrm{~cm}^{2} / W, W_{0}=2 \mu \mathrm{m}, U=10 \mu \mathrm{J}, \tau=10 \mathrm{~ns}, \ldots$, with it the optical tweezer is brokendown. To reduce the Kerr effect and relating self-focusing 
effect, it is necessary to choice the laser beam in accordance with the nonlinear coeficient of refractive index of the medium embedding the particle, so that the trapping condition $m_{n}>1$ is satisfied always.

\section{REFERENCES}

[1] A. Ashkin, Phys. Rev. Lett. 24 (1970) 156-159.

[2] A. Ashkin, J. M. Dziedzic, J.E. Bjorkholm, S. Chu, Opt. Lett. 11 (1986) 288-290.

[3] S. C. Kuo, M. P. Sheetz, Trends Cell Biol. 2 (1992) 116-118.

[4] F. M. Fazal and S. M. Block, Nature Photonics 5 (2011) 318-321.

[5] A. A. Ambardekar, Y. Q. Li, Opt. Lett. 30 (2005) 1797-1799.

[6] J. L. Deng, Q. Wei, Y. Z. Wang, Y. Q. Li, Opt. Express. 13 (2006) 3673-3680.

[7] C. L. Zhao, L. G. Wang, X. H. Lu, Phys. Let. A363 (2006) 502-506.

[8] L.G. Wang et al., Opt. Lett. 32 (2007) 1393-1395.

[9] H. Q. Quy, M. V. Luu, IWPESA, Nha Trang, Sep.10-14 (2008) pp.181-186.

[10] H. Kress, Ernest H. K. Stelzer, G. Griffiths, and A. Rohrbach, Phys. Rev. E71 (2005) 061927.

[11] E.V. Lyubin, M. D. Khokhlova, M. N. Skryabina, and A. A. Fedyanin, J. of Biomedical Optics 17 (2012) 101510.

[12] Q. Q. Ho, J. Phys. Scien. and Appl. 2(9) (2012) 301-305.

[13] Q. Q. Ho and V. N. Hoang, J. Phys. Scien. and Appl. 2(10) (2012) 414-419.

[14] R. W. Boyd, "Nonlinear Optics," Academic Press. Inc. (1992) 261.

[15] B. E. A. Saleh and M.C. Teich, "Fundamentals of Photonics," A Willey-Interscience Publication, John Willey \& Sons, Inc., New York (1991) 92-93.

[16] Keir C. Neuman and Steven M. Block, Review of Science Intruments 75(9) (2004) 2787-2809.

[17] H. Q. Quy, M. V. Luu, H. D. Hai, D. Zhuang, Chinese Optics Letters 8 (2010) 332-334. 